\title{
Use of Scanning Electron Microscopy and Energy Dispersive Spectroscopy to Correlate the Arrangement of Starch with Resistance to Maize Weevil (Coleoptera: Curculionidae) in Sorghum Grain
}

\author{
M. W. Pendleton, ${ }^{*}$ B. B. Pendleton, ${ }^{* *}$ E. A. Ellis, ${ }^{*}$ G. C. Peterson, ${ }^{* * *}$ Fernando M. Chitio, ${ }^{* * * *}$ \\ and Suhas Vyavhare** \\ * Microscopy and Imaging Center, Texas A\&M University, Biological Sciences Building Room \\ Number 119, Mail Stop 2257, College Station, TX 77843-2257 \\ ** Department of Agricultural Sciences, P.O. Box 60998, West Texas A\&M University, Canyon, \\ TX 79016-0001 \\ *** Texas AgriLife Research, Texas A\&M University, Route 3, Box 219, Lubbock, TX 79401 \\ **** Nampula Research Center, IIAM, P.O. Box 36, Ave. Josina Machel, Nampula, Mozambique
}

The maize weevil, Sitophilus zeamais Motschulsky, is an important pest of grain of stored sorghum, Sorghum bicolor (L.) Moench [1]. Weevil larvae and adults feed on kernels in the field and in storage. Chitio [2] determined the resistance of 20 genotypes of sorghum grains to the weevils. He placed $5 \mathrm{~g}$ of grain of each sorghum genotype into a vial with three female and two male maize weevils. Numbers of live and dead weevils, damage, and weight loss were determined for each sorghum genotype for 105 days. In this study, seven of the genotypes were examined to determine whether depth of starch from the seed coat was related to resistance to maize weevils.

A JEOL JSM-6400 scanning electron microscope (SEM) (15 KeV, $15 \mathrm{~mm}$ working distance) was used to show secondary images of weevil mouthparts and the sorghum kernels in cross section. An energy dispersive spectroscopy (EDS) detector on the SEM produced EDS plots and maps using PGT (Bruker) Spirit software. To prepare the kernels for SEM imaging, grains of seven genotypes of sorghum were cut with a razor (1 mm depth), fixed in $2.5 \%$ Glutaraldehyde - $1 \%$ Acrolein in HEPES buffer ( $\mathrm{pH} 7.3$ ), followed by 1\% Osmium in HEPES buffer ( $\mathrm{pH} 7.3$ ). Dehydration by methanol was done at 5\% steps, followed by three changes of Hexamethyldisilazane (HMDS). After fracturing along the cut area, the grains were mounted on stubs and coated with iodine vapor using the method of Ellis and Pendleton [3] to locate starch. A Cressington 308R evaporative coater (Ted Pella, Inc., Redding, CA) was used to apply a coating of $35 \mathrm{~nm}$ of carbon to the kernels before SEMEDS.

The EDS detector was used to determine the elements in grain of the seven genotypes of sorghum. To locate the area of the kernel that contained starch, iodine was applied as a vapor [3] over the cross section of each grain. Iodine vapor binds with starch. A representative EDS plot of the area of a cross section near the seed coat of Segaolane sorghum (Fig. 1) included iodine, carbon, silicon, osmium, and chlorine. Secondary SEM images of grain of each of the seven genotypes of sorghum tested were produced (Segaolane sorghum example Fig. 2A). The EDS detector produced a map of the same areas of each kernel that contained iodine (bound to starch) (Segaolane sorghum Fig. 2B). The distance from the seed coat to the band of concentrated iodine (and starch) was measured for each genotype and plotted on the EDS maps (Segaolane sorghum Fig. 2B).

A positive correlation in these seven genotypes was demonstrated as an increase in depth of a band of concentrated iodine (with bound starch) measured from the seed coat and the degree of resistance to damage by the maize weevil. This measurement may be compared to that of new genotypes of sorghum to predict their resistance to maize weevil [4]. 
References

[1] G.L. Teetes et al, Tex. Ag. Ex. Station Report B-1371. College Station, Texas, 1981.

[2] F.M. Chitio, M.S. thesis, West Texas A\&M University, Canyon, Texas, 2004.

[3] E.A. Ellis and M.W. Pendleton, Micros. Today 15 No. 3 (2007) 44.

[4] This research was supported in part by the Sorghum, Millet and Other Grains Collaborative Research Support Program (INTSORMIL CRSP) sponsored by the United States Agency for International Development.

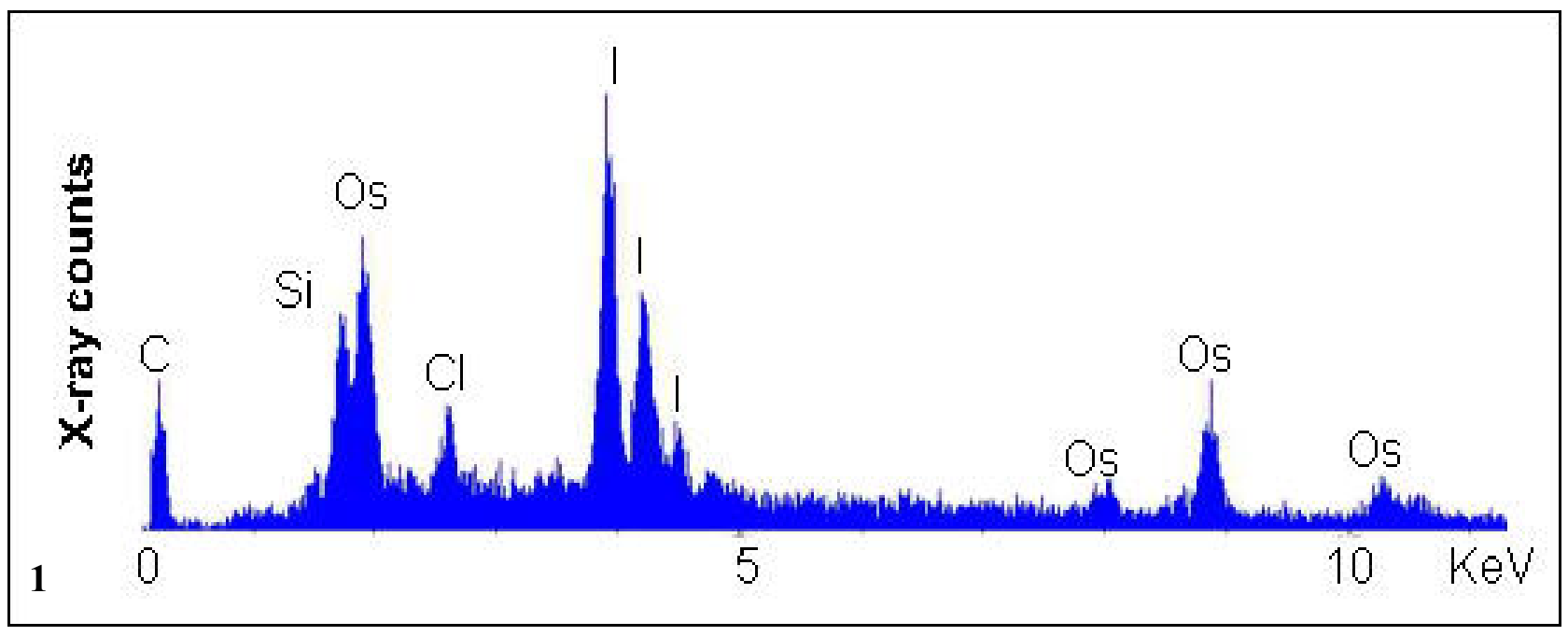

Fig. 1. Energy Dispersive Spectroscopy (EDS) spectrum of Segaolane sorghum kernel. Peaks are elements detected including osmium added as a fixative and iodine added to locate starch.
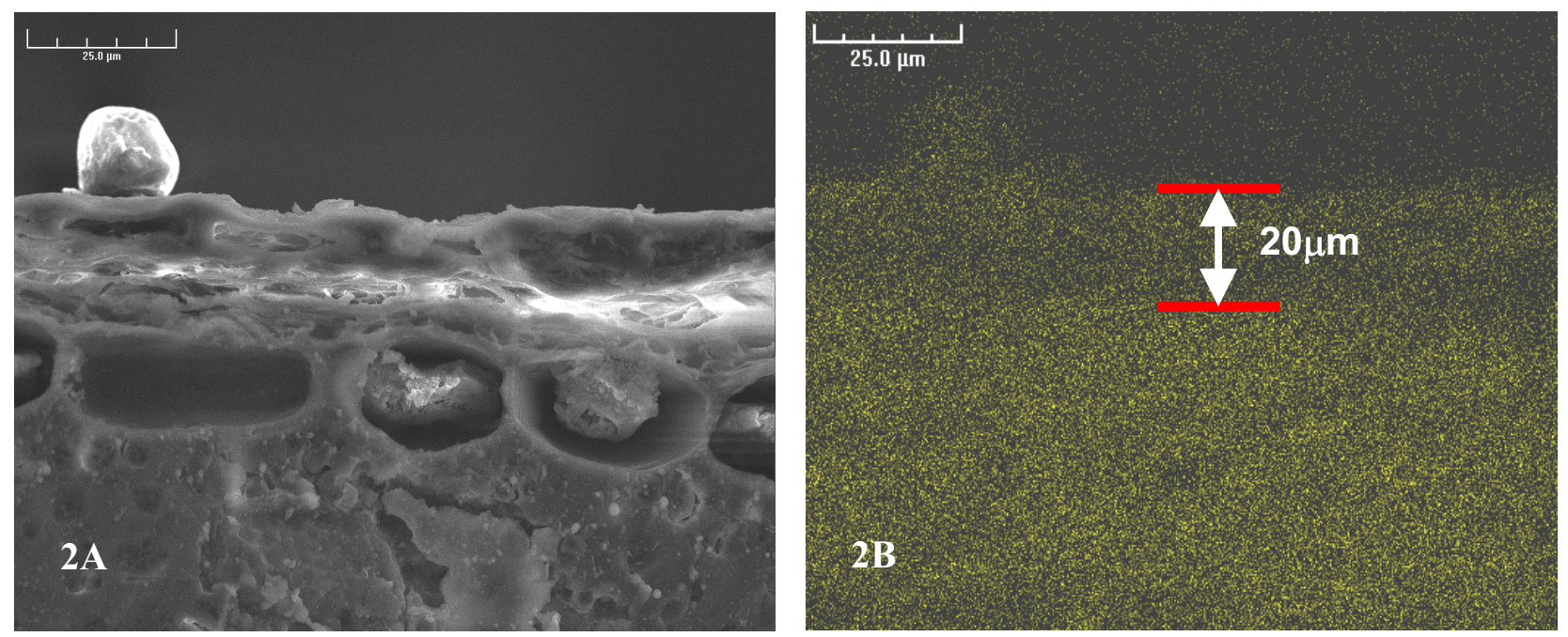

Fig. 2A. Segaolane secondary electron image, bar $=25 \mu \mathrm{m}$. Fig. 2B. Segaolane iodine L $\alpha$ orbital EDS map with measurement from seed coat to band of starch concentration area, bar $=25 \mu \mathrm{m}$. 